\title{
Rotamer Modelling of $\mathrm{Cu}(\mathrm{II})$ Spin Labels Based on the Double-Histidine Motif
}

\author{
Shreya Ghosh ${ }^{1} \cdot$ Sunil Saxena ${ }^{1}$ (D) Gunnar Jeschke ${ }^{2}$ (D)
}

Received: 18 May 2018 / Revised: 19 July 2018 / Published online: 16 August 2018

(C) The Author(s) 2018

\begin{abstract}
Spin labels attached to two residues of a protein chain have less conformational flexibility than those attached to a single residue and thus lead to a narrower spatial distribution of the unpaired electron. The case of $\mathrm{Cu}^{2+}$ labels based on the doublehistidine (dHis) motif is of particular interest, as it combines the advantage of precise localization of the unpaired electron with a labelling scheme orthogonal to the more common cysteine-based labelling. Here, we introduce an approach for in silico spin labelling of a protein by dHis motifs and $\mathrm{Cu}^{2+}$ complexes of iminodiacetic acid or nitrilotriacetic acid. We discuss a computerized scan for native histidine pairs that might be prone to bind such $\mathrm{Cu}^{2+}$ complexes and spin-labelling site pair scans that can identify suitable double mutants for labelling. Predicted distance distributions between two $\mathrm{Cu}^{2+}$ labels are compared to experimental distance distributions. We also test the hypothesis that elastic network modelling of conformational transitions with $\mathrm{Cu}^{2+}$-dHis labels can provide more accurate structural models than with nitroxide labels.
\end{abstract}

\section{Introduction}

The combination of pulsed dipolar spectroscopy techniques, such as double electron electron resonance (DEER), also known as PELDOR [1, 2], double-quantum coherence (DQC) [3, 4], relaxation-induced dipolar modulation enhancement (RIDME) $[5,6]$, or the single-frequency techniques for refocusing (SIFTER) dipolar couplings $[7,8]$, with site-directed spin labelling $[9,10]$ has developed into an important tool in structural biology [11-14], especially for proteins and protein complexes with

Sunil Saxena

sksaxena@pitt.edu

Gunnar Jeschke

gjeschke@ethz.ch

1 Department of Chemistry, University of Pittsburgh, Pittsburgh, PA 15260, USA

2 Department of Chemistry and Applied Biosciences, ETH Zürich, Vladimir-Prelog-Weg 2, 8093 Zurich, Switzerland 
intermediate order of backbone conformation [15]. Most applications use nitroxide spin labels attached to a single cysteine residue, such as the methanethiosulfonate spin label (MTSSL), which has a moderately flexible linker between the backbone and the nitroxide moiety. Bifunctional attachment to two cysteine residues, as in the Rx spin label that features two methanethiosulfonate groups [16], leads to a much narrower spatial distribution of the unpaired electron as demonstrated by molecular dynamic simulations [17]. However, binding to both residues is not always achieved. Furthermore, given that the removal of all native cysteines in a protein is not always feasible, alternative site-directed labelling approaches are in demand. For some problems in structure determination, spectroscopically orthogonal labelling by two different paramagnetic centres is required [18], which has also led to an interest in metal-ion based labels.

In this context, pulsed electron paramagnetic resonance (EPR) spectroscopic techniques involving $\mathrm{Cu}^{2+}$ have been developed [19-28]. Strategies involving $\mathrm{Cu}^{2+}$ bound to native binding sites in the protein have proven to be an important technique for structural determination of proteins [19, 29-33]. In cases where proteins do not have endogenous $\mathrm{Cu}^{2+}$-binding sites, several spin labelling techniques have been developed which site-specifically incorporate $\mathrm{Cu}^{2+}$ to a protein using chelating tags [34]. While these tags show high affinity to $\mathrm{Cu}^{2+}$ and require only a single mutation in the protein (specifically, cysteine), they are flexible and can provide distributions that are at best the same as MTSSL. Another alternative labelling method for $\mathrm{Cu}^{2+}$ involves the use of two histidine residues, placed strategically to bind $\mathrm{Cu}^{2+}$ [35] known as the double histidine (dHis) motif. In addition this motif uses an $i, i+4$ placement of histidine residues on an $\alpha$-helix or $i, i+2$ for a $\beta$-sheet. Even though each $\mathrm{dHis}-\mathrm{Cu}^{2+}$ requires mutation of two residues, the motif shows almost five times narrower distance distribution than MTSSL, indicating that it is a much rigid spin label. However, a major limitation of this motif when using just $\mathrm{Cu}^{2+}$ is that $\mathrm{Cu}^{2+}$ shows poor selectivity to the dHis. Thus, $\mathrm{Cu}^{2+}$ may coordinate unspecifically to other residues in the protein.

To overcome this limitation, the $\mathrm{Cu}^{2+}$ ion was chelated by ligands such as iminodiacetic acid (IDA) [35, 36] or nitrilotriacetic acid (NTA) [37]. Using $\mathrm{Cu}^{2+}-$ IDA or $\mathrm{Cu}^{2+}-\mathrm{NTA}$ increases the binding specificity of the complex to the dHis site only, while reducing unspecific binding. The dHis motif, being highly rigid, provides a distance distribution which can be almost five times narrower than that obtained using standard nitroxide spin labels such as methanethiosulfonate spin label $[35,37]$. This development is important because side chain flexibility is a major limitation for the precision and accuracy of structural models that can be obtained with restraints from MTSSL-based spin labelling. Because of the high flexibility of MTSSL, the distance distribution is dominated by the motion of the spin label rather than the actual backbone fluctuations [38] unless backbone disorder is substantial [15].

To further develop this methodology, systematic methods are required that use the $\mathrm{Cu}^{2+}$-based distance restraints to provide protein structure and conformational dynamics. So far, nitroxide-based distance restraints along with the multiscale modeling of macromolecular systems (MMM) software [39, 40] have been used to provide such information. MMM generates a library of rotamers based on the conformational space of the spin label. Distance restraints along with restraints generated 
from the elastic network model (ENM) in MMM [41, 42] can then be used to create models of protein structure and conformational dynamics. Due to the high rigidity, distance restraints obtained with the dHis motif with $\mathrm{Cu}^{2+}-\mathrm{IDA} / \mathrm{NTA}$ are expected to improve the accuracy of such models as compared to nitroxide spin labels, as the initial study had shown that the flexible linker leads to an accuracy loss [41]. Hence, there is a need to incorporate dHis-based distance restraints into the MMM software. Such an implementation should also allow to test whether native pairs of histidine residues may be prone to binding $\mathrm{Cu}^{2+}-\mathrm{IDA}$ or $\mathrm{Cu}^{2+}-\mathrm{NTA}$ and should be able to suggest site pairs for labelling in the spirit of the site scan feature [43] for nitroxide labels attached to a single cysteine residue. To this end, in this work we have generated histidine rotamer libraries and structural models for the $\mathrm{Cu}^{2+}$ complexes formed with a dHis motif and either of the two chelating ligands, IDA or NTA. Experimental distance constraints obtained on dHis-Cu ${ }^{2+}-$ IDA/NTA along with MMM simulations can thus provide direct insight into the protein backbone structure and conformation.

This paper is structured as follows: In Sect. 1, we discuss the general approach for modelling attachment of a bifunctional label to a pair of residues. In Sect. 2, we explain our hypothesis on the structure of the formed complexes, which is based on available crystal structures, general knowledge of coordination preferences of $\mathrm{Cu}^{2+}$, and density functional theory computations. In Sect. 3, we describe the implementation of labelling, visualization, and site scans into MMM. In Sect. 4, we compare distance distributions predicted by our approach to those measured previously on the B1 immunoglobulin-binding domain of protein G (GB1) [17-19] and on human glutathione $S$-transferase A1-1 protein (huGSTA1-1) [37, 44]. In Sects. 5 and 6, we test by simulations for a set of known structural transitions whether restraints based on dHis- $\mathrm{Cu}^{2+}$ labelling can indeed be expected to improve on the accuracy of structural models as compared to restraints obtained with MTSSL.

\section{Modelling Approach}

\subsection{Attachment of Bifunctional Labels}

A bifunctional label attaches to the sidechains of two amino acid residues if these are in a spatial arrangement that satisfies the steric requirements of the label. Usually, these requirements will be rather strict since low conformational variability of the label is a desired property. It is thus necessary for the two sidechains to adopt a rather well-defined binding pose. This requirement may still allow for some variability in the $C^{\alpha}-C^{\alpha}$ distance and relative local frame orientation of the residues, since even native sidechains such as the ones of cysteine or histidine can adopt different rotameric states. The first step of modelling the attachment thus consists of testing whether any rotamer pairs of the binding residues fulfil the spatial requirements for label attachment. We shall define these requirements for $\mathrm{dHis}_{-} \mathrm{Cu}^{2+}$ coordination in the next Section.

The problem of finding suitable rotamer combinations for the two histidine residues can be solved with the same rotamer library approach that we have previously 
introduced for modelling single-site labels [40]. For generation of the histidine rotamer library, we employed our newer approach based on a Monte Carlo sampling of conformations and hierarchical clustering [42], except that no softening of the Lennard-Jones potentials was required and an ensemble of 50,000 low-energy conformations was generated and clustered. The initial structure of histidine was optimized with the MMFF94 force field in Chem3D (Perkin Elmer). The histidine sidechain has only two rotatable bonds with three energy minima for the torsion angle $\chi_{1}$ and four minima for the torsion angle $\chi_{2}$ (see Fig. 1), giving 12 rotamers. Hence, for each site pair only 144 rotamer pairs need to be tested, which makes site scans feasible even for large proteins. The histidine rotamer library is included with the name HCU_298K_UFF_12_r5 in MMM 2018.

\subsection{Modelling of Copper Coordination}

The complex of $\mathrm{Cu}^{2+}$ with a dHis site and iminodiacetic acid (IDA) was originally expected to have an approximately octahedral geometry with two equatorial nitrogen ligands from the dHis site, an equatorial nitrogen and an equatorial oxygen ligand from IDA and an axial oxygen ligand from IDA [35]. The final axial position is thought to be occupied by water. A corresponding crystal structure [45] was downloaded from the Cambridge Structural Database (CSD) as a CIF file (accession number 650681) and converted with Mercury to a Sybyl MOL2 file. In this structure (Fig. 1), the coordinated nitrogen atoms of the two imidazole ligands have distances of 1.987 and $2.001 \AA$ from the $\mathrm{Cu}^{2+}$ ion, whereas the nitrogen atom of the IDA ligand has a distance of $2.085 \AA$ and the equatorial oxygen atom of the IDA ligand has a distance of $1.965 \AA$. The axial oxygen atom of IDA has a distance of $2.225 \AA$. The $\mathrm{N}^{\delta}-\mathrm{Cu}-\mathrm{N}^{\delta^{\prime}}$ angle for the two imidazole ligands is $92.1^{\circ}$ and the $\mathrm{N}^{\delta}-\mathrm{N}^{\delta^{\prime}}$ distance is $2.872 \AA$. Because of the two very similar $\mathrm{Cu}-\mathrm{N}$ distances, we term this coordination mode of IDA 'symmetric'. It is modelled by the conformer library dHis_Cu_ IDA_sym that can be accessed with the label name IDS.

The structure was edited in Accelrys Discovery Studio to the cis-[iminodiacetato$\left.\kappa^{3} \mathrm{~N}, \mathrm{O}, \mathrm{O}^{\prime}\right]$ bis $\left(1 \mathrm{H}\right.$-imidazole- $\left.\mathrm{N}^{3}\right)$ copper(II) complex $\left(\mathrm{N}^{3}\right.$ is $\left.\mathrm{N}^{\delta}\right)$ and the MOL2 file
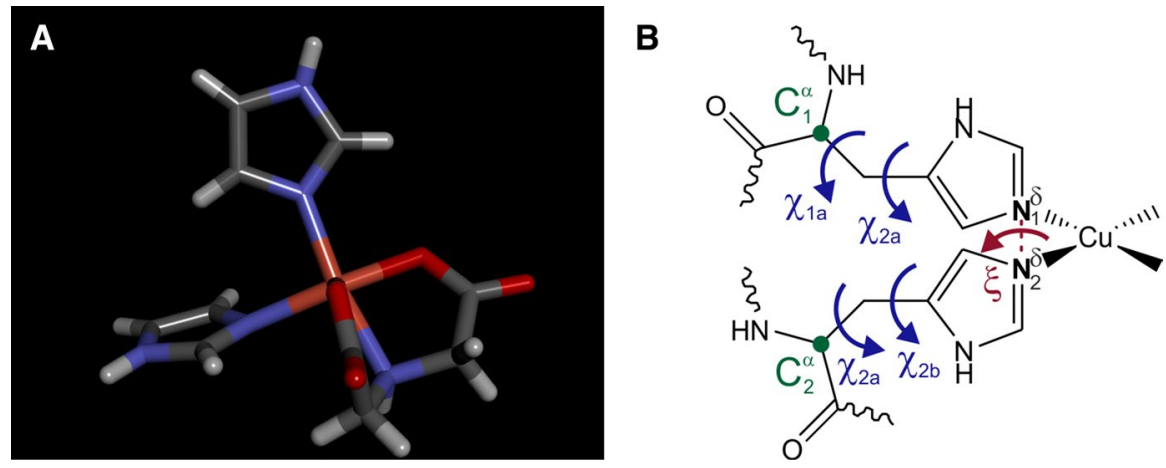

Fig. 1 a Stick model of the crystal structure of the bis(imidazole)-IDA $\mathrm{Cu}^{2+}$ complex. b Definition of torsion angles for the two histidine residues and for copper orientation with respect to their two $\mathrm{N}^{\delta}$ atoms 
of the edited structure was converted to a Cartesian coordinate file using Chem3D (Perkin-Elmer). It was then optimized on restricted Kohn-Sham level with the BP86 density functional, the def2-SVP basis set for all atoms, and the conductor-like polarizable continuum solvent model for water in ORCA 4.0.1 [46]. In the optimized structure (Fig. 2), we find lengths of the $\mathrm{Cu}-\mathrm{N}$ dative bonds to the two imidazole nitrogens of 2.012 and $2.216 \AA$, respectively, an $\mathrm{N}^{\delta}-\mathrm{Cu}-\mathrm{N}^{\delta^{\prime}}$ angle of $101.4^{\circ}$, and an $\mathrm{N}^{\delta}-\mathrm{N}^{\delta^{\prime}}$ distance of $3.273 \AA$ between the two imidazole nitrogens. In this structure, one of the two imidazoles acts as an axial ligand, whereas the square plane is of a $2 \mathrm{~N} 2 \mathrm{O}$ type with distances of 2.011 and $2.014 \AA$ to the two coordinated oxygen atoms and $2.057 \AA$ to the coordinated nitrogen atom of IDA. We term this coordination mode 'asymmetric'. It is modelled by the conformer library dHis_Cu_IDA_ asym that can be accessed with the label name IDA. This is the default library for the IDA ligand that we use throughout this paper.

The complex of $\mathrm{Cu}^{2+}$ with a dHis site and nitrilotriacetic acid (NTA) was originally expected to have an approximately octahedral geometry with two equatorial nitrogen ligands from the dHis site, an equatorial nitrogen and an equatorial oxygen ligand from NTA and two axial oxygen ligands from NTA [37]. A crystal structure with the same number and type of coordinated atoms and at least similar geometry [47] has accession code 927203 in the CSD and features a 2,2'-bipyridine ligand instead of two imidazole ligands. Indeed, the two nitrogen atoms of the bipyridine form strong dative bonds with $\mathrm{Cu}-\mathrm{N}$ distances of 1.989 and $2.005 \AA$. Ligand geometry restrains the $\mathrm{N}-\mathrm{N}$ distance to $2.597 \AA$, leading to a $\mathrm{N}-\mathrm{Cu}-\mathrm{N}$ angle of $81.1^{\circ}$. One of the coordinating oxygen atoms of the NTA ligand lies in the $\mathrm{N}-\mathrm{Cu}-\mathrm{N}$ plane and forms a strong dative bond with a length of $1.936 \AA$. All other coordinating atoms of NTA are out of plane.

The ligand was edited in Accelrys Discovery Studio using the imidazole bond lengths and angles observed in the DFT-optimized structure of the IDA complex. The monoanionic NTA complex was then optimized on restricted Kohn-Sham level with the BP86 density functional, the def2-SVP basis set for all atoms, and the

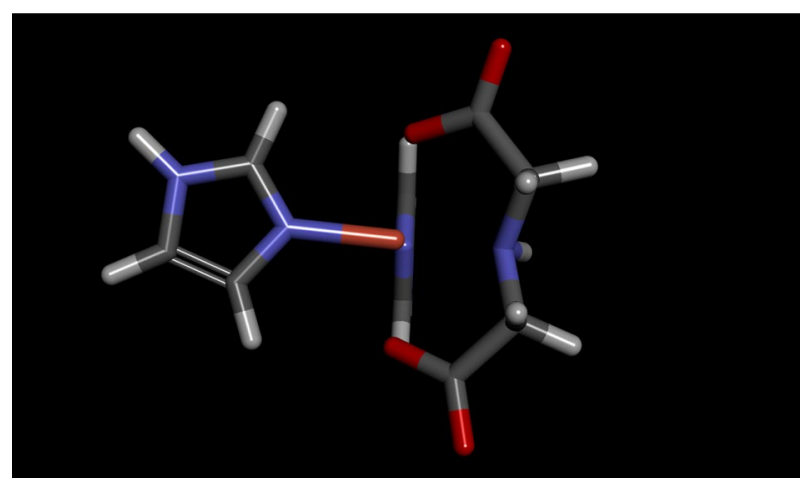

Fig. 2 Stick model of the DFT-optimized structure of the bis(imidazole)-IDA $\mathrm{Cu}^{2+}$ complex. View perpendicular to the equatorial plane. The second imidazole ligand in the axial position is perpendicular to the image plane and situated below the $\mathrm{Cu}^{2+}$ atom. Differences in the $\mathrm{Cu}^{2+}$ position compared to symmetric binding are minor 
conductor-like polarizable continuum solvent model for water in ORCA 4.0.1 [46]. In the optimized structure, only one of the imidazole ligands was found to be coordinated. This result was reproduced for two somewhat different initial geometries.

We then decided to link the two imidazole ligands, as they are linked in a protein context. To that end we edited them to 4-methylene-imidazoles and inserted a $-\mathrm{CH}_{2}-\mathrm{CH}=\mathrm{CH}-\mathrm{CH}=\mathrm{CH}-$ link between the methylene carbons. To overcome difficulties with self-consistent field convergence in the vicinity of our initial geometry, we pre-optimized the structure without invoking the conductor-like polarizable continuum solvent model and with Fermi-like occupation number smearing at a temperature of $5000 \mathrm{~K}$. Even in this case, we observed dissociation of one of the imidazole ligands from the complex. In fact, the bipyridine complex, which is part of a coordination polymer in the crystal structure, does not retain its octahedral coordination geometry when DFT optimized in isolation. The optimized geometry is a square pyramid with the two nitrogen atoms of the bipyridine ligand and two oxygens of NTA in equatorial positions and the third oxygen in the axial position at a distance of $2.122 \AA$. The nitrogen atom of NTA is not coordinated $(2.592 \AA$, not in the other axial position).

Therefore, we gave up on the hypothesis that the dHis motif and NTA together occupy all six coordination positions of $\mathrm{Cu}^{2+}$ and based optimization on the complex of a keto-bridged bismethylimidazole-NTA ligand with $\mathrm{Cu}^{2+}$ with CSD accession number 167295 [48]. Coordination geometry is again a square pyramid with the two methylimidazole nitrogen atoms and two oxygen atoms of NTA taking the equatorial positions and the nitrogen of NTA taking the axial position (Fig. 3). The third carboxylic group of NTA is not coordinated. We first optimized the complex, then severed the keto bridge and rotated the methylimidazole rings out of plane and optimized again. In the final structure, we find lengths of the $\mathrm{Cu}-\mathrm{N}$ dative bonds to the two imidazole nitrogens of 2.033 and $2.034 \AA$, and $\mathrm{N}-\mathrm{Cu}-\mathrm{N}$ angle of $93.2^{\circ}$, and an $\mathrm{N}-\mathrm{N}$ distance of $2.954 \AA$ between the two imidazole nitrogens. The equatorial oxygen atoms are at a distances of 1.986 and $2.027 \AA$ and the axial nitrogen is at a distance of $2.360 \AA$. This is again

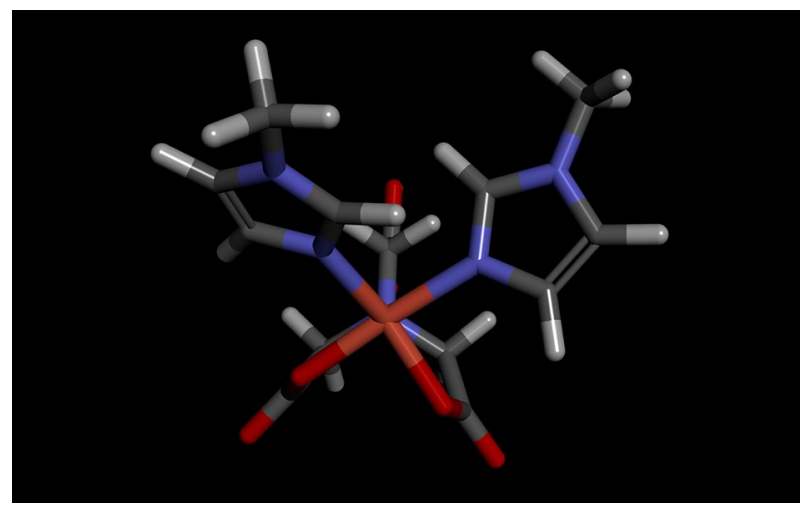

Fig. 3 Stick model of the DFT-optimized structure of the bis(methylimidazole)-NTA $\mathrm{Cu}^{2+}$ complex. View perpendicular to the equatorial plane. The nitrogen atom of the NTA ligand in the axial position is situated below the $\mathrm{Cu}^{2+}$ atom 
a symmetric coordination mode, which we model by the conformer library dHis_Cu_ NTA_sym that can be accessed with the label name NTA.

\subsection{Modelling of the $\mathrm{Cu}^{2+}$ Site}

We assume that a dHis motif will bind $\mathrm{Cu}^{2+}-\mathrm{IDA}$ or $\mathrm{Cu}^{2+}-\mathrm{NTA}$ if a combination of histidine rotamers exists that poses the $\mathrm{N}^{3}\left(\mathrm{~N}^{\delta}\right)$ atoms of the two histidine sidechains at a distance allowing for coordination. The $\mathrm{Cu}^{2+}$ coordination polyhedron is rather plastic $[49,50]$ as is also apparent from the results of the DFT computations described above. These results suggest that $\mathrm{N}^{\delta}-\mathrm{N}^{\delta^{\prime}}$ distances between 2.6 and $3.2 \AA$ can be accommodated. We apply this range for all three ligand conformer libraries. In the first step, we select all combinations of histidine rotamers at the two sites whose $\mathrm{N}^{\delta}-\mathrm{N}^{\delta^{\prime}}$ distance $d_{\mathrm{NN}}$ falls within this range.

We further assume that the polar (IDA) or charged (NTA) $\mathrm{Cu}^{2+}$ complexes will tend to point away from the protein surface. If we choose the $x$-axis of a protein-fixed local frame along the $\mathrm{N}^{3}-\mathrm{N}^{3 \prime}$ vector and the centre of mass of the protein in the $x y$-plane with a coordinate $y<0$, our first guess for the $\mathrm{Cu}^{2+}$ position is thus in the $x y$-plane with a coordinate $y>0$. As the origin of the frame we take the centre of the $\mathrm{N}^{\delta}-\mathrm{N}^{\delta^{\prime}}$. This local frame is also defined for the complex structure, so that the initial guess for the $\mathrm{Cu}^{2+}$ and ligand (IDA or NTA) position can be obtained by superimposition of the two local frames.

The geometry of the $\mathrm{Cu}^{2+}$ site is thus fixed, except for rotation of all non-protein atoms about the $\mathrm{N}^{\delta}-\mathrm{N}^{\delta^{\prime}}$ axis. We assume an empirical cosine potential for this rotation with an energy minimum at rotation angle $\xi=0$ of the geometry described above, where the $\mathrm{Cu}^{2+}$ ion is farthest away from the centre of mass of the protein. The barrier height $V_{\xi}$ of this torsion potential, which has a maximum at $\xi=180^{\circ}$, can be fitted to reproduce experimentally observed distance distributions. To the energy of this potential, we add the Lennard-Jones interaction energy of the non-protein atoms with the protein atoms as in our rotamer library approach [40]. For a soft torsion potential with a barrier height $V_{\xi}<1 \mathrm{~kJ} / \mathrm{mol}$, the distribution of $\xi$ is mainly restrained by clashes of the ligand with protein atoms. For a hard torsion potential with $V_{\xi}>100 \mathrm{~kJ} / \mathrm{mol}$, coordination induces significant strain on local protein structure. Given the fact that at a 1:1 ratio of $\mathrm{Cu}^{2+}$-IDA to dHis sites complete attachment is not observed [36], we consider it as unlikely that such coordination can induce substantial strain.

We discretize the $\xi$ torsion about the $\mathrm{N}^{3}-\mathrm{N}^{3^{\prime}}$ axis to keep in line with our approach of modelling a label by a small number of conformations. Discretization in steps of $5^{\circ}$ ensures that the $\mathrm{Cu}^{2+}$ coordinates of the $\xi$ rotamers are sufficiently close spaced.

\section{Implementation and Usage in MMM}

\subsection{Site Scans}

In spin-labelling site scans [43] we consider only residue pairs within the same chain. Such site scans can still correspond to a considerable computational effort, 
since for a chain with $n$ residues we need to test $n(n-1) / 2$ site pairs. This quadratic scaling of the computational expense of a site scan with peptide chain length reduces to a nearly linear scaling by considering that $\mathrm{N}^{\delta}-\mathrm{N}^{\delta^{\prime}}$ distances between 2.6 and $3.2 \AA$ can be realized only if the $C^{\alpha}-C^{\alpha}$ distance is shorter than $10 \AA$. Although such distances can be realized for residue pairs $(i, i+\Delta i)$ with large $\Delta i$, we consider only residues whose numbers differ by $1 \leq \Delta i \leq 5$. As explained above, canonical site pairs have $\Delta i=4$ for two sites in an $\alpha$ helix and $\Delta i=2$ for two sites in a $\beta$ sheet. The site scan report is stored as a text file and specifies, for each site pair that is expected to coordinate $\mathrm{Cu}^{2+}$, the two potential coordinating residues, their $\mathrm{C}^{\alpha}-\mathrm{C}^{\alpha}$ distance, the number of significantly populated conformers, the partition function, the rootmean-square deviation of the $\mathrm{Cu}^{2+}$ position from its mean, and the $\mathrm{Cu}$-backbone distance, defined as the distance of the $\mathrm{Cu}^{2+}$ ion from the midpoint of the $\mathrm{C}^{\alpha}-\mathrm{C}^{\alpha}$ vector of the two residues. The report is automatically opened in the MMM report editor.

For finding pairs of native histidine residues that are susceptible to binding a $\mathrm{Cu}^{2+}$-IDA or $\mathrm{Cu}^{2+}-\mathrm{NTA}$ complex, we allow for any sequence distance $\Delta i$ within the same peptide chain. This functionality was tested on a few crystal structures and an unexpected potential-binding motif was found between the remote histidines 106 and 203 in bacterial L-asparaginase crystal structure 1JSL [51]. Whether this protein indeed binds to $\mathrm{Cu}^{2+}$-IDA was not checked experimentally, but the possibility of such coordination should be checked if a structure of a protein is available.

\subsection{Visualization}

In contrast to our previous implementation for nitroxide spin labels, the implementation for $\mathrm{Cu}^{2+}$-dHis labels stores information on labelling separately from the structure, i.e., the internal representation of the protein is not changed. While this has some advantages, for instance, when testing alternative labels in the same MMM session, it prevents visualization of all-atom models of the attached $\mathrm{Cu}^{2+}$ complex. Instead, we opted for visualization of the distribution of $\mathrm{Cu}^{2+}$ positions, which leads to a clearer representation (Fig. 4).

\subsection{Usage}

In MMM 2018, $\mathrm{Cu}^{2+}$-dHis site scans, labelling, and visualization are accessible only via the command line. After the $\mathrm{Cu}^{2+}$ position distributions have been predicted, distance distributions and DEER time-domain data can be computed as for nitroxide labels in the DEER window. The use of $\mathrm{Cu}^{2+}$-dHis restraints in elastic network modelling of conformational transitions is explained in Sect. 5.

For any protein of small or moderate size, we recommend working with the blscan command, which has the syntax

\section{blscan address label outfile [native] [torsionpot],}

where address denotes a chain address (Example: $(A)$ for chain $\mathrm{A}$ of the current structure), label can be IDA for the asymmetric attachment of the IDA ligand (default), IDS for the symmetric attachment of the IDA ligand, or NTA for the NTA 

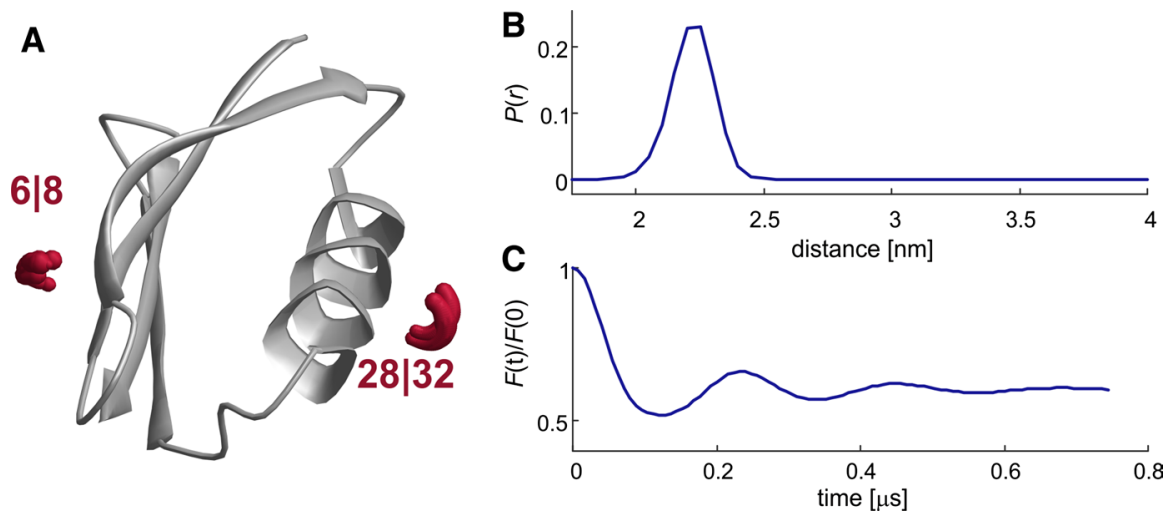

Fig. 4 Predicted spatial distribution of $\mathrm{Cu}^{2+}$-IDA in the $\mathrm{GB} 1$ double pair mutant $6 \mathrm{H} / 8 \mathrm{H} / / 28 \mathrm{H} / 32 \mathrm{H}$ based on crystal structure PDB 1PGA [52]. a Visualization of the $\mathrm{Cu}^{2+}$ distribution. b Distance distribution. c DEER form factor

ligand. The parameter outfile is the file name for the site scan report (default: bilabel_site_scan), which is stored in the current directory, but can be saved to any other directory from the report editor window. The parameter native can be native, true, or false, where the first two choices instruct the program to search for pairs of native histidine pairs that can coordinate $\mathrm{Cu}^{2+}$, irrespective of the sequence distance. This parameter defaults to false. The parameter torsionpot denotes the torsion potential in $\mathrm{kJ} / \mathrm{mol}$, is optional and defaults to $10 \mathrm{~kJ} / \mathrm{mol}$ (see Sect. 4).

If the dHis sites of interest are already known and the protein is large, it may be more convenient to label individual dHis sites with the bilabel command with the syntax

\section{bilabel address 1 address 2 label [torsionpot].}

The parameters are analogous, except that address 1 and address 2 are addresses of the two residues that together make up the dHis site. These residues can be of any type, mutation to histidine is automatically computed. Although the command allows for the two residues to be situated in different peptide chains, we discourage such labelling except for methodological work.

Finally, the $\mathrm{Cu}^{2+}$ positions can be visualized with the standard show command with modified address syntax

show address $1 \mid$ address 2 label color.

Again, address 1 and address 2 are addresses of the two residues that together make up the dHis site, which are separated without space by the vertical line separator. The argument 'label' must be written verbatim, it does not denote the type of label. The color argument is optional and can be either a scalable vector graphics color name (crimson was used for Fig. 4) or a red-green-blue (RGB) triple of values in the range from 0 to 1 .

Other standard functionality of MMM is available after either the blscan or bilabel command has been performed. In particular, $\mathrm{Cu}^{2+}-\mathrm{dHis}$ labels can be selected in 
the DEER window of MMM and distance distributions (Fig. 4b) as well as DEER form factors (Fig. 4c) can be predicted and compared to experimental data that were processed with the DeerAnalysis software.

The new features are implemented into MMM from version 2018 onwards. MMM is freely available as an open-source package for Matlab (The MathWorks Inc, Natick, MA, USA) at http://www.epr.ethz.ch/software.html.

\section{Comparison to Experimental Data}

\subsection{B1 Immunoglobulin Binding Domain of Protein G (GB1)}

GB1 is a 56-amino acid B1 immunoglobulin-binding domain of protein G. GB1 is a simple model system used in protein-folding studies and other applications because of its high-thermal stability and well resolved structures obtained from X-ray crystallography, NMR, EPR and other methods [20, 53, 54]. Hence, using the dHis-Cu ${ }^{2+}$ motif on GB1 helps to predict the accuracy of the motif for determining structural constraints and conformational changes in the protein in solution. In this work, we have compared data from MMM simulations with the experimental data previously obtained on two different mutant constructs of GB1 [36, 37]. For both the tetramutant GB1 constructs, the dHis site at the $\alpha$-helix was positioned at $28 \mathrm{H} / 32 \mathrm{H}$. For the $\beta$-sheet, one of the construct had the $\mathrm{dHis}$ site at $6 \mathrm{H} / 8 \mathrm{H}$, while the other had it at positions $15 \mathrm{H} / 17 \mathrm{H}$. While $\mathrm{Cu}^{2+}$-IDA was used only for $6 \mathrm{H} / 8 \mathrm{H} / 28 \mathrm{H} / 32 \mathrm{H}$ [36], $\mathrm{Cu}^{2+}-\mathrm{NTA}$ was used with both the mutants [37]. The $6 \mathrm{H} / 8 \mathrm{H} / 28 \mathrm{H} / 32 \mathrm{H}$ tetramutant GB1 is shown in Fig. 5a.

The experimental most probable distance for the tetramutant $6 \mathrm{H} / 8 \mathrm{H} / 28 \mathrm{H} / 32 \mathrm{H}$ GB1 spin labeled with $\mathrm{Cu}^{2+}-$ IDA was $2.47 \mathrm{~nm}$ with a standard deviation of $0.08 \mathrm{~nm}$ [36]. For performing MMM simulations, we considered five different PDB files as well as different torsion potentials on the spin label (Fig. 6). We considered the coordination mode of IDA to be 'symmetric' such that the two nitrogen atoms of the imidazole ligands are in the equatorial plane of $\mathrm{Cu}^{2+}$. The default torsion potential used by MMM is $10 \mathrm{~kJ} / \mathrm{mol}$. An increase in the torsion potential leads to a narrower distance distribution while a broader distance distribution is favored for a lower torsion potential. For most cases, a torsion potential of $0.1 \mathrm{~kJ} / \mathrm{mol}$ leads to a larger distribution width than the experimental one and the most probable distance was short $(\sim 2.16 \mathrm{~nm})$. On increasing the potential to $10 \mathrm{~kJ} / \mathrm{mol}$, the distribution width was comparable to that of the experiment with the most probable distance still being $2.5 \AA$ shorter than the experimental one. Further increase in the torsion potential to $100 \mathrm{~kJ} / \mathrm{mol}$ only leads to a marginal improvement. Keeping in mind that the crystal structures 4WH4, 1PGA and 2QMT have a resolution of $2.2 \AA, 2.07 \AA$ and $1.05 \AA$, respectively, we can consider the simulated most probable distance to be within error.

We see similar agreement between simulated and experimental results for the tetramutants $6 \mathrm{H} / 8 \mathrm{H} / 28 \mathrm{H} / 32 \mathrm{H}-\mathrm{GB} 1$ and $15 \mathrm{H} / 17 \mathrm{H} / 28 \mathrm{H} / 32 \mathrm{H}-\mathrm{GB} 1$ with $\mathrm{Cu}^{2+}-\mathrm{NTA}$ (Figs. 7, 8). A discrepancy was, however, observed when spin labeling the PDB files $4 \mathrm{WH} 4$ and 1PGA at sites 6,8 (for a potential of $100 \mathrm{~kJ} / \mathrm{mol}$ ) and 15, 17, 

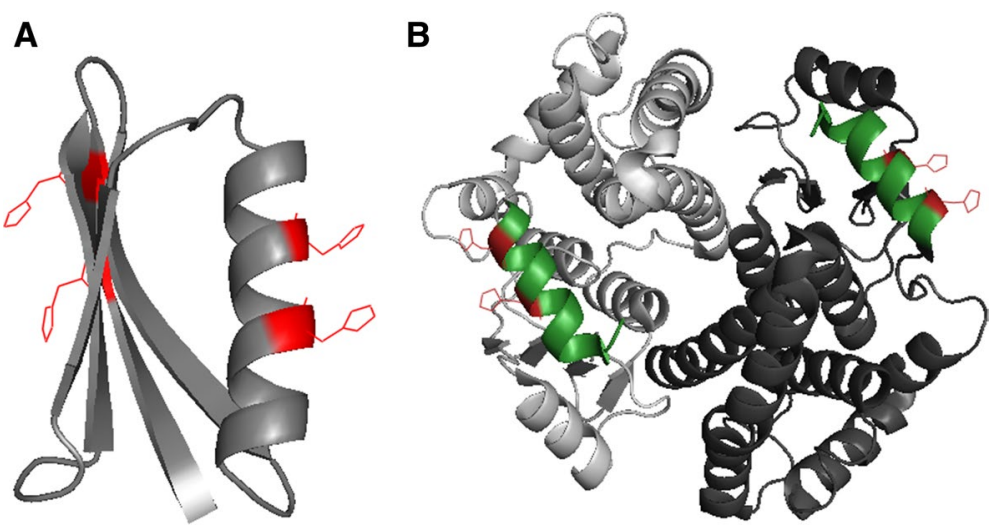

Fig. 5 a Tetramutant GB1 with dHis at sites $6 \mathrm{H} / 8 \mathrm{H}$ in the $\beta$-sheet and $28 \mathrm{H} / 32 \mathrm{H}$ in the $\alpha$-helix. The residues in red indicate the histidine of the dHis. b Dimeric huGSTA1-1 with a dHis site $(211 \mathrm{H} / 215 \mathrm{H})$ at each subunit. The two subunits are shown by light and dark gray while the Histidine residues are shown in red. The helices in green represent $\alpha 9$ (color figure online)

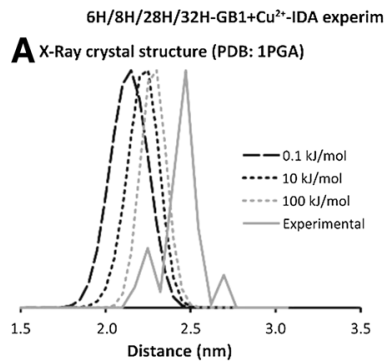

D solid-state NMR structure (PDB: 2LGI)

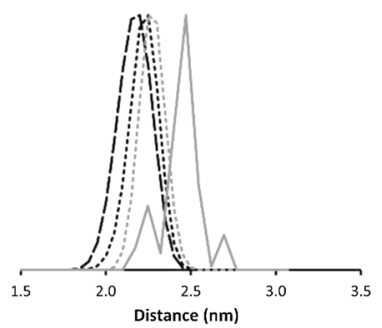

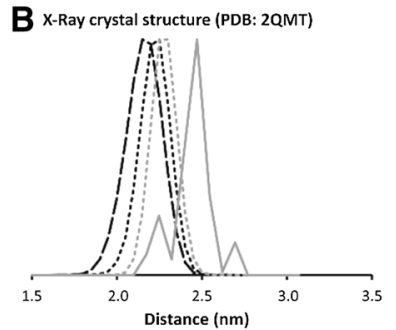

E solution NMR structure (PDB: 1GB1)

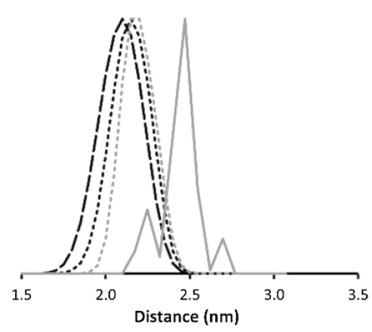

Fig. 6 MMM simulations compared to experimental data of $6 \mathrm{H} / 8 \mathrm{H} / 28 \mathrm{H} / 32 \mathrm{H}-\mathrm{GB} 1$ with $\mathrm{Cu}^{2+}-\mathrm{IDA}$ for five different PDB structures. Simulations were also performed at different torsion potentials $V_{\xi}(0.1,10$ and $100 \mathrm{~kJ} / \mathrm{mol}$ ). For most cases, while a potential of $0.1 \mathrm{~kJ} / \mathrm{mol}$ leads to a larger distribution width, a potential of $10 \mathrm{~kJ} / \mathrm{mol}$ leads to a distribution width similar to that of the experimental. Further increase to $100 \mathrm{~kJ} / \mathrm{mol}$ did not lead to any significant change

respectively. We were not able to spin label the abovementioned sites with $\mathrm{Cu}^{2+}-\mathrm{NTA}$ in silico. The likely reason is that the ligand clashes with other protein atoms at the minimum of the assumed torsion potential. 


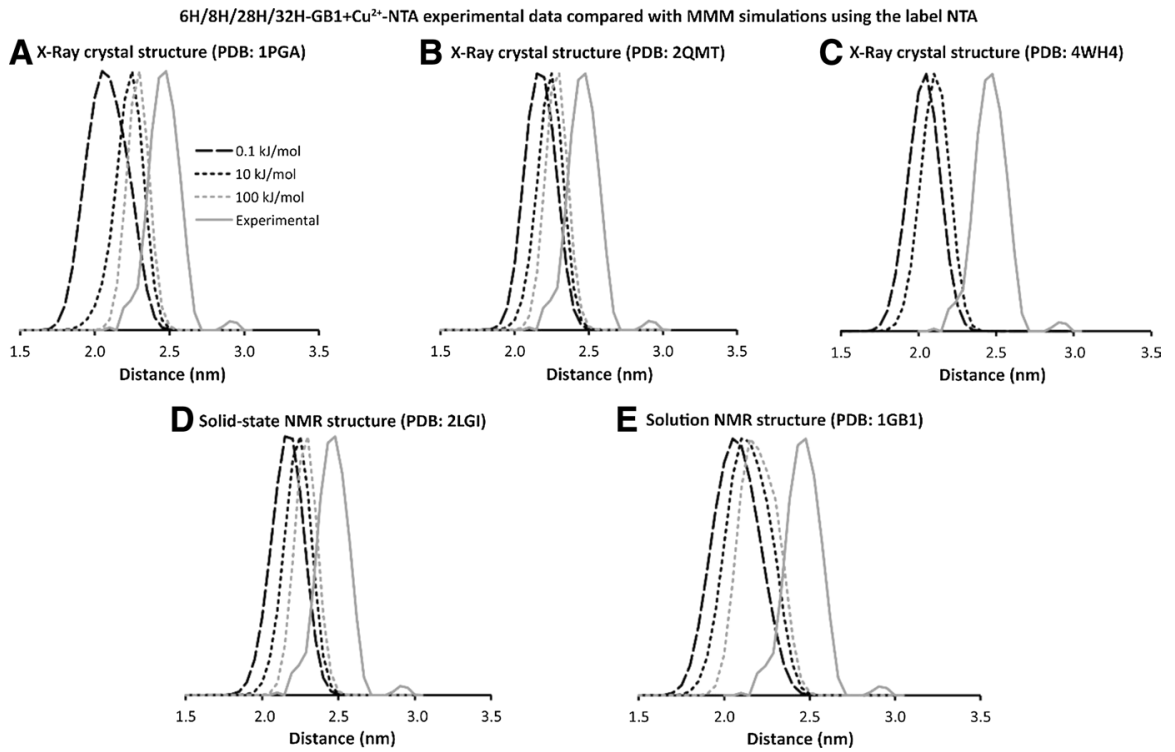

Fig. 7 MMM simulations and DEER data for $6 \mathrm{H} / 8 \mathrm{H} / 28 \mathrm{H} / 32 \mathrm{H}-\mathrm{GB} 1+\mathrm{Cu}^{2+}-\mathrm{NTA}$ showed similar agreement as mentioned for $\mathrm{Cu}^{2+}-\mathrm{IDA}$

A $x$-Ray crystal structure (PDB: 4WH4)

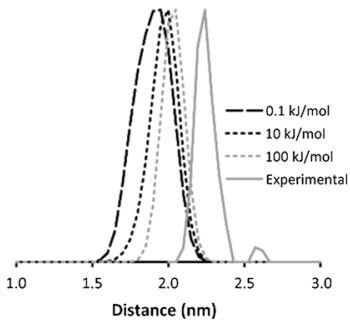

$15 \mathrm{H} / 17 \mathrm{H} / 28 \mathrm{H} / 32 \mathrm{H}-\mathrm{GB} 1+\mathrm{Cu}^{2+}-\mathrm{NTA}$

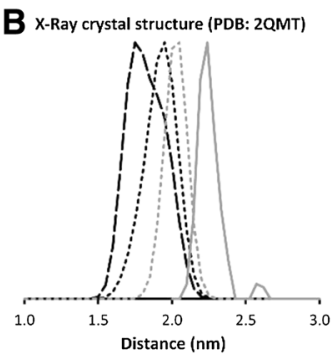

C Solid-state NMR structure (PDB: 2LGI)

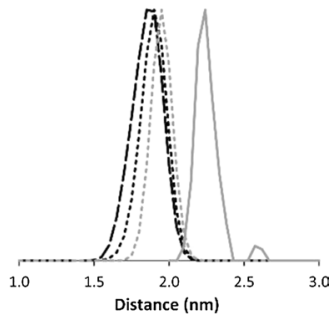

Fig. 8 Comparing MMM simulations with DEER data on $15 \mathrm{H} / 17 \mathrm{H} / 28 \mathrm{H} / 32 \mathrm{H}-\mathrm{GB} 1+\mathrm{Cu}^{2+}-\mathrm{NTA}$ showed results similar to that of $6 \mathrm{H} / 8 \mathrm{H} / 28 \mathrm{H} / 32 \mathrm{H}$ with $\mathrm{Cu}^{2+}-\mathrm{NTA}$

In general, the most probable distance appears to be within the resolution of the PDB structures. That said, the simulated most probable distances are systematically shorter than the experimental ones for both IDA and NTA labels in GB1. Variation of the predicted mean distance $6 \mathrm{H} / 8 \mathrm{H} / 28 \mathrm{H} / 32 \mathrm{H}-\mathrm{GB} 1$ among the 60 conformations in the NMR structural ensemble does not exceed $0.9 \AA$. The remaining discrepancy between predictions and experimental data may then indicate that the $\mathrm{Cu}^{2+}$ ion is slightly further away from the backbone than suggested by our histidine rotamer library and our complex structures. This issue needs to be re-examined once more data from proteins with known structure become available. 


\subsection{Human Glutathione S-transferase A1-1 Protein (huGSTA1-1)}

Glutathione $S$-transferase (GST) superfamily consists of a class of proteins that are diverse in their amino acid sequence. All GSTs play an important role in the cellular defence mechanism for a large number of species. The vital function of GSTs is their ability to catalyse the nucleophilic attack by glutathione (GSH) on the electrophilic atoms of the xenobiotic substrates [55]. The electrophiles then have a deactivated reactive center which prevents the xenobiotic substrates from reacting with crucial cellular proteins and nucleic acids.

The human $\alpha$-GST (huGSTA1-1) is a 222-residue homodimer protein that is believed to exist in two major conformations in the ligand-free state [56]. Conformations of the huGSTA1-1 in the ligand-bound state has been well resolved using X-ray crystallography and NMR [57-59]. However, in the ligand-free state, the conformation of a critical 11-residue helix at the C-terminal, called $\alpha 9$, has only recently been identified [44]. Recently, DEER was performed on the unliganded huGSTA1-1 [44]. Distance measurements using MTSSL spin labels on the $\alpha 9$ helix displayed a bimodal distribution which was consistent with two distinct conformations of the protein. These distances along with MMM simulations were used to generate a model of the $\alpha 9$ conformation in the unliganded state. In addition, distance measurements were performed by incorporating a dHis site $(\mathrm{K} 211 \mathrm{H} / \mathrm{E} 215 \mathrm{H})$ in each subunit within the dimeric protein (cf. Fig. 5b). The resultant DEER data using $\mathrm{Cu}^{2+}$-IDA showed bimodal distance distribution, which is consistent with the presence of two conformations of the $\alpha 9$ helix in ligand-free huGSTA1-1.

Figure 9 shows the dHis-based distance distribution obtained in the absence of the ligand. Previously, we identified the larger distance as being consistent with the $\alpha 9$ conformation observed in the crystal structure of the ligand-bound enzyme (PDB: 1K3L). Indeed, labeling this structure (PDB: 1K3L) by dHis in MMM generates a distance distribution that closely matches the experimental distance. For the shorter distance we used the structure obtained by a combination of MTSSL distances and MMM simulations [44]. On performing MMM simulations on the same conformer but using dHis- $\mathrm{Cu}^{2+}$, we observed the most probable distance to be around $4.0 \mathrm{~nm}$ for a $0.1 \mathrm{~kJ} / \mathrm{mol}$ torsion potential and $4.1 \mathrm{~nm}$ for torsion potentials of 10 and $100 \mathrm{~kJ} / \mathrm{mol}$. Given that the structure of this conformer was generated using only MTSSL-based distance constraints, we expect the simulated most probable dHis-based distance to differ from that of experimental $(\sim 3.6 \mathrm{~nm})$. Nevertheless, the modeled distance is within the experimental distribution.

In the case of huGSTA1-1, the experimental distance distributions are much broader than the ones predicted by our modelling. This cannot be traced back to an underestimate of the spatial distribution of the label with respect to the backbone in our approach, since even with a flat torsion potential, the experimentally observed widths cannot be matched. The broad distance distributions for huGSTA1-1 thus indicate some conformational flexibility of the peptide backbone in solution that is suppressed by packing effects upon crystallization. While preliminary, these results underscore the potential of dHis-based distances to measure backbone flexibility.

$\mathrm{Cu}^{2+}-\mathrm{Cu}^{2+}$ distance distributions simulated for a fixed backbone conformation have full widths at half height widths around $2 \AA$ that vary little and agree well with 


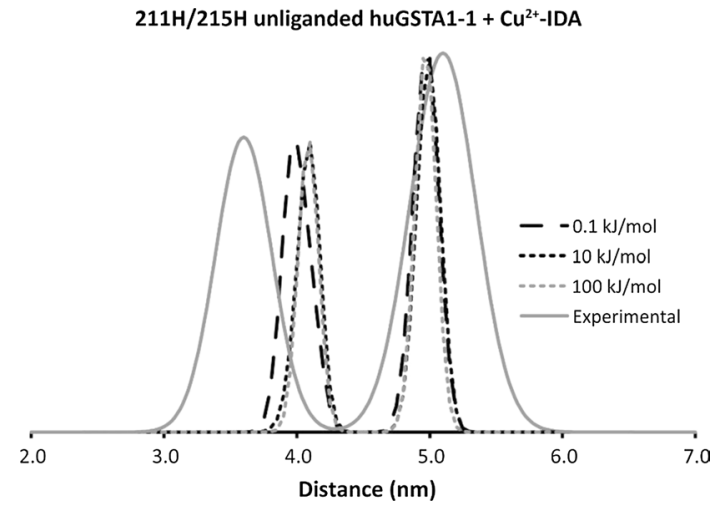

Fig. 9 Experimental data of unliganded 211H/215H huGSTA1-1 with $\mathrm{Cu}^{2+}$-IDA compared to MMM simulations showing both the possible conformations. MMM simulations using $\mathrm{dHis}-\mathrm{Cu}^{2+}$ were performed on the liganded GST structure (PDB: 1K3L) and showed good agreement with the longer distance of the unliganded protein. Simulations were also performed on the previously obtained model which was based on MTSSL distances and X-ray crystal structure constraints [44]. The simulations resulted in a most probable distance of $\sim 4 \mathrm{~nm}$ compared to the experimental $3.6 \mathrm{~nm}$. This difference can be attributed to the fact that the model used for simulation was roughly based on sparse experimental MTSSL data

the experimental widths measured on GB1. Backbone flexibility beyond this width should thus be visible in experimental data. Given a sufficient number of restraints, it can be simulated with the approach described in [60] which is implemented in MMM and has been adapted for $\mathrm{Cu}^{2+}$-dHis labels.

\section{Accuracy of Modelling Conformational Change by an Elastic Network Model Approach}

\subsection{Basic Considerations and Implementation}

Here, we consider the modelling of a conformational transition of a protein based on a structure of the initial state and a moderate number of distance restraints for the final state, supported by an anisotropic elastic network model (ENM) [61]. Previous research has demonstrated that with distances between spin labels, the accuracy of the model for the final state, quantified by a fractional coverage $f$ of the conformational change, is worse than when $C^{\alpha}-C^{\alpha}$ distances for the same site pairs are used [41]. This loss in accuracy presumably results from the conformational flexibility of the spin label sidechain. Since this flexibility is smaller for $\mathrm{Cu}^{2+}$-dHis labelling, we hypothesized that a higher fractional coverage can be obtained with $\mathrm{Cu}^{2+}-\mathrm{dH}$ is labels as compared to MTSSL. This hypothesis is best tested by simulations on state pairs where both the initial and final structure are experimentally known $[41,61]$. From the set of 18 such pairs used in our previous studies $[41,62]$, we focused on cases where a fractional coverage $f>0.5$ was found with 20 optimal $C^{\alpha}-C^{\alpha}$ distance restraints, indicating that the ENM 
reasonably well models the transition. We use the optimized algorithm based on equipartitioning of energy between the normal modes of the ENM with 20 restraints, an initial active space dimension $B_{0}=20$, and the edENM parametrization of the ENM [62]. For the two label cases, we determined the mean fractional coverage for an ensemble of 20 models of the final structure computed with different distance restraint sets where each individual restraint was set by a normally distributed random number with the mean and standard deviation of the simulated distance distribution for this label pair.

The choice of labelling sites is based on optimization for MTSSL sites [54]. Following site scans for $\mathrm{Cu}^{2+}$-dHis-IDA, we selected histidine pairs that overlapped with the optimal MTSSL sites or had a maximum sequence distance of two residues from them. This strategy excluded four potential test cases (1GGG/1WDN, 1BNC/1DV2, 1B7T/1DFK, 1N0V/1N0U), since in these cases corresponding dHis sites could not be found for all MTSSL sites in both the initial and final structure. We still preferred this strategy to independent optimization of the sites for the individual labels, since for the latter strategy the results are influenced not only by conformational variability of the label but also by differences in the linear combination of the normal modes of the ENM.

The results for the remaining six test cases are summarized in Table 1. In five cases, fractional coverage indeed improves when going from MTSSL to a dHisbased label. In the case of 1L5B/1L5E we observe a slight decrease. We note that the mean coverage for an ensemble obtained with varying restraint sets has an uncertainty. For the smallest protein, which incidentally is the 1L5B/1L5E case, we computed six 20-membered ensembles each for MTSSL and $\mathrm{Cu}^{2+}$-dHis-IDA. The mean fractional coverage for all ensembles is 0.570 for MTSSL and 0.562 for $\mathrm{Cu}^{2+}$-dHis-IDA, with standard deviations of 0.062 and 0.016 , respectively. The larger standard deviation for MTSSL is expected because of the broader distance distributions. For the test case 1AON/1OEL the optimal set of MTSSL restraint sites performs poorly compared to an optimal set of $\mathrm{C}^{\alpha}$ restraint sites $(f=0.566)$, even if $\mathrm{C}^{\alpha}$ restraints are used at the optimal MTSSL sites. Altogether, we can conclude that the expected improvement in accuracy when using the more rigid dHis-based labels is borne out in the simulations for most cases.

Table 1 Fractional mean coverage of conformational transitions in elastic network modelling with simulated restraints

\begin{tabular}{lllllll}
\hline & 1L5B/1L5E & 2LAO/1LST & 1OMP/1ANF & 1EPS/1G6S & 1AON/1OEL & 1SU4/1T5S \\
\hline $\mathrm{C} \alpha$ & 0.783 & 0.793 & 0.468 & 0.780 & 0.162 & 0.696 \\
$\mathrm{MTSSL}$ & 0.613 & 0.518 & 0.224 & 0.444 & 0.190 & 0.498 \\
$\mathrm{Cu}^{2+}$-dHis-IDA & 0.575 & 0.578 & 0.320 & 0.613 & 0.214 & 0.602 \\
\hline
\end{tabular}

For MTSSL and $\mathrm{Cu}^{2+}$-dHis-IDA mean values for an ensemble of 20 models are given 


\section{Conclusion}

In-silico spin labelling for labels that attach to two residues is feasible with a computational effort that allows for site scans of large proteins. The approach is based on a rotamer library for the sidechains of the native attachment residues and a conformer library for the actual label. It was implemented for $\mathrm{Cu}^{2+}-\mathrm{dHis}$ labelling with the auxiliary ligands IDA and NTA. Despite some uncertainty on the structure of the complexes, the approach is robust for $i, i+4$ surface-exposed dHis sites in $\alpha$-helices and for $i, i+2$ surface-exposed dHis sites in $\beta$-sheets, since for given histidine coordinates, the $\mathrm{Cu}^{2+}$ coordinates depend only weakly on exact complex structure.

Tests of the prediction of most probable interspin distance suggest an accuracy of about 2-3 $\AA$, not much worse than the resolution of the crystal structures on which the predictions were based. While this accuracy is similar to that observed for the best MTSSL rotamer libraries, prediction of the width and shape of the distribution is much more accurate for $\mathrm{Cu}^{2+}$-dHis-based labels than for MTSSL. Furthermore, the more rigid $\mathrm{Cu}^{2+}$ labels lead to a better accuracy in modeling of conformational transitions based on simulated label-to-label distance restraints and an elastic network model. Although we did not test by either experiments or simulations whether such improvement is also found for other types of modelling structure from label-label distance distributions restraints, this appears likely. For this reason, we believe that further experimental and computational research into $\mathrm{Cu}^{2+}-\mathrm{dHis}$ labels is desirable. Such research will profit from the in-silico labelling approach introduced here.

Acknowledgements This work was supported by the Swiss National Science Foundation (200020_169057 to G.J.) and the National Science Foundation (NSF MCB-1613007 and NSF MRI1725678 to S.S.). We thank Dr. Matthew Lawless for useful discussions.

Open Access This article is distributed under the terms of the Creative Commons Attribution 4.0 International License (http://creativecommons.org/licenses/by/4.0/), which permits unrestricted use, distribution, and reproduction in any medium, provided you give appropriate credit to the original author(s) and the source, provide a link to the Creative Commons license, and indicate if changes were made.

\section{References}

1. A.D. Milov, K.M. Salikhov, M.D. Shchirov, Fiz. Tverd. Tela 23, 975 (1981)

2. M. Pannier, S. Veit, A. Godt, G. Jeschke, H.W. Spiess, J. Magn. Reson. 142, 331 (2000)

3. S. Saxena, J.H. Freed, J. Chem. Phys. 107, 1317 (1997)

4. P.P. Borbat, J.H. Freed, Chem. Phys. Lett. 313, 145 (1999)

5. L.V. Kulik, S.A. Dzuba, I.A. Grigoryev, Y.D. Tsvetkov, Chem. Phys. Lett. 343, 315 (2001)

6. S. Milikisyants, F. Scarpelli, M.G. Finiguerra, M. Ubbink, M. Huber, J. Magn. Reson. 201, 48 (2009)

7. G. Jeschke, M. Pannier, A. Godt, H.W. Spiess, Chem. Phys. Lett. 331, 243 (2000)

8. P. Schöps, P.E. Spindler, A. Marko, T.F. Prisner, J. Magn. Reson. 250, 55 (2015)

9. W.L. Hubbell, D.S. Cafiso, C. Altenbach, Nat. Struct. Biol. 7, 735 (2000)

10. S.A. Shelke, S.T. Sigurdsson, Eur. J. Org. Chem. 2012, 2291 (2012)

11. O. Schiemann, T.F. Prisner, Q. Rev. Biophys. 40, 1 (2007)

12. P.P. Borbat, J.H. Freed, Methods Enzymol. 423, 52 (2007) 
13. H.S. Mchaourab, P.R. Steed, K. Kazmier, Structure 19, 1549 (2011)

14. G. Jeschke, Annu. Rev. Phys. Chem. 63, 419 (2012)

15. G. Jeschke, Emerg. Top. Life Sci. 2, 9 (2018)

16. M.R. Fleissner, M.D. Bridges, E.K. Brooks, D. Cascio, T. Kálai, K. Hideg, W.L. Hubbell, Proc. Natl. Acad. Sci. USA 108, 16241 (2011)

17. S.M. Islam, B. Roux, J. Phys. Chem. B 119, 3901 (2015)

18. B. Joseph, V.M. Korkhov, M. Yulikov, G. Jeschke, E. Bordignon, J. Biol. Chem. 289, 3176 (2014)

19. M. Ji, S. Ruthstein, S. Saxena, Acc. Chem. Res. 47, 688 (2014)

20. Z. Yang, M. Ji, T.F. Cunningham, S. Saxena, Methods Enzymol. 563, 459 (2015)

21. I.M.C. van Amsterdam, M. Ubbink, G.W. Canters, M. Huber, Angew. Chem. Int. Ed. 42, 62 (2003)

22. E. Narr, A. Godt, G. Jeschke, Angew. Chem. Int. Ed. 41, 3907 (2002)

23. Z. Yang, J. Becker, S. Saxena, J. Magn. Reson. 188, 337 (2007)

24. B.E. Bode, J. Plackmeyer, T.F. Prisner, O. Schiemann, J. Phys. Chem. A 112, 5064 (2008)

25. J.E. Lovett, A.M. Bowen, C.R. Timmel, M.W. Jones, J.R. Dilworth, D. Caprotti, S.G. Bell, L.L. Wong, J. Harmer, Phys. Chem. Chem. Phys. 11, 6840 (2009)

26. Z. Yang, D. Kise, S. Saxena, J. Phys. Chem. B 114, 6165 (2010)

27. J.S. Becker, S. Saxena, Chem. Phys. Lett. 414, 248 (2005)

28. S. Ruthstein, M. Ji, P. Mehta, L. Jen-Jacobson, S. Saxena, J. Phys. Chem. B 117, 6227 (2013)

29. G.E. Merz, P.P. Borbat, A.J. Pratt, E.D. Getzoff, J.H. Freed, B.R. Crane, Biophys. J. 107, 1669 (2014)

30. J.H. van Wonderen, D.N. Kostrz, C. Dennison, F. MacMillan, Angew. Chem. Int. Ed. 52, 1990 (2013)

31. Z. Yang, M.R. Kurpiewski, M. Ji, J.E. Townsend, P. Mehta, L. Jen-Jacobson, S. Saxena, Proc. Natl. Acad. Sci. USA 109, E993 (2012)

32. E.G.B. Evans, M.J. Pushie, K.A. Markham, H.-W. Lee, G.L. Millhauser, Structure 24, 1057 (2016)

33. H.E.M. Christopher, W.M. Kay, A. Richard Cammack, R.W. Evans, J. Am. Chem. Soc. 129, 4868 (2007)

34. T.F. Cunningham, M.D. Shannon, M.R. Putterman, R.J. Arachchige, I. Sengupta, M. Gao, C.P. Jaroniec, S. Saxena, J. Phys. Chem. B 119, 2839 (2015)

35. T.F. Cunningham, M.R. Putterman, A. Desai, W.S. Horne, S. Saxena, Angew. Chem. Int. Ed. 54, 6330 (2015)

36. M.J. Lawless, S. Ghosh, T.F. Cunningham, A. Shimshi, S. Saxena, Phys. Chem. Chem. Phys. 19, 20959 (2017)

37. S. Ghosh, M.J. Lawless, G.S. Rule, S. Saxena, J. Magn. Reson. 286, 163 (2018)

38. J.L. Sarver, J.E. Townsend, G. Rajapakse, L. Jen-Jacobson, S. Saxena, J. Phys. Chem. B 116, 4024 (2012)

39. G. Jeschke, Protein Sci. 27, 76 (2018)

40. Y. Polyhach, E. Bordignon, G. Jeschke, Phys. Chem. Chem. Phys. 13, 2356 (2011)

41. G. Jeschke, J. Chem. Theory Comput. 8, 3854 (2012)

42. E.A. Suturina, D. Häussinger, K. Zimmermann, L. Garbuio, M. Yulikov, G. Jeschke, I. Kuprov, Chem. Sci. 8, 2751 (2017)

43. Y. Polyhach, G. Jeschke, Spectroscopy 24, 651 (2010)

44. M.J. Lawless, J.R. Pettersson, G.S. Rule, F. Lanni, S. Saxena, Biophys. J. 114, 592 (2018)

45. M.P. Brandi-Blanco, M.M. de Benavides-Giménez, J.M. González-Pérez, D. ChoquesilloLazarte, Acta Crystallogr. Sect. E 63, m1678 (2007)

46. F. Neese, Wiley Interdiscip. Rev. Comput. Mol. Sci. 2, 73 (2012)

47. N. Kumari, M.A. Hasan, B.D. Ward, L. Mishra, Ind. Eng. Chem. Res. 52, 15007 (2013)

48. C.J. Burns, L.D. Field, T.W. Hambley, T. Lin, D.D. Ridley, P. Turner, M.P. Wilkinson, ARKIVOC vii, 157 (2001)

49. J. Gaăzo, I.B. Bersuker, J. Garaj, M. Kabešová, J. Kohout, H. Langfelderová, M. Melník, M. Serator, F. Valach, Coord. Chem. Rev. 19, 253 (1976)

50. B. Murphy, B. Hathaway, Coord. Chem. Rev. 243, 237 (2003)

51. K. Aghaiypour, A. Wlodawer, J. Lubkowski, Biochim. Biophys. Acta Protein Struct. Mol. Enzymol. 1550, 117 (2001)

52. T. Gallagher, P. Alexander, P. Bryan, G.L. Gilliland, Biochemistry 33, 4721 (1994) 
53. I. Sengupta, P.S. Nadaud, J.J. Helmus, C.D. Schwieters, C.P. Jaroniec, Nat. Chem. 4, 410 (2012)

54. V. Muñoz, P.A. Thompson, J. Hofrichter, W.A. Eaton, Nature 390, 196 (1997)

55. R.N. Armstrong, Chem. Res. Toxicol. 10, 2 (1997)

56. M.T. Honaker, M. Acchione, W. Zhang, B. Mannervik, W.M. Atkins, J. Biol. Chem. 288, 18599 (2013)

57. Y. Zhan, G.S. Rule, Biochemistry 43, 7244 (2004)

58. E. Grahn, M. Novotny, E. Jakobsson, A. Gustafsson, L. Grehn, B. Olin, D. Madsen, M. Wahlberg, B. Mannervik, G.J. Kleywegt, Acta Crystallogr. Sect. D Biol. Crystallogr. 62, 197 (2006)

59. I. Le Trong, R.E. Stenkamp, C. Ibarra, W.M. Atkins, E.T. Adman, Proteins Struct. Funct. Bioinform. 48, 618 (2002)

60. G. Jeschke, Proteins Struct. Funct. Bioinform. 84, 544 (2016)

61. W. Zheng, B.R. Brooks, Biophys. J. 90, 4327 (2006)

62. G. Jeschke, Z. Phys. Chem. 226, 1395 (2012) 